\title{
Finite Conductivity Effects in Electrostatic Force Microscopy on Thin Dielectric Films: A Theoretical Model
}

\author{
E. Castellano-Hernández ${ }^{1}$ and G. M. Sacha ${ }^{2}$ \\ ${ }^{1}$ Instituto de Ciencia de Materiales de Madrid, CSIC, Campus de Cantoblanco, 28049 Madrid, Spain \\ ${ }^{2}$ Departamento de Ingeniería Informática, Escuela Politécnica Superior, Universidad Autónoma de Madrid, 28049 Madrid, Spain \\ Correspondence should be addressed to G. M. Sacha; sacha.gomez@uam.es
}

Received 7 May 2015; Revised 26 August 2015; Accepted 30 August 2015

Academic Editor: Da-Ren Hang

Copyright (C) 2015 E. Castellano-Hernández and G. M. Sacha. This is an open access article distributed under the Creative Commons Attribution License, which permits unrestricted use, distribution, and reproduction in any medium, provided the original work is properly cited.

\begin{abstract}
A study of the electrostatic force between an Electrostatic Force Microscope tip and a dielectric thin film with finite conductivity is presented. By using the Thomas-Fermi approximation and the method of image charges, we calculate the electrostatic potential and force as a function of the thin film screening length, which is a magnitude related to the amount of free charge in the thin film and is defined as the maximum length that the electric field is able to penetrate in the sample. We show the microscope's signal on dielectric films can change significantly in the presence of a finite conductivity even in the limit of large screening lengths. This is particularly relevant in determining the effective dielectric constant of thin films from Electrostatic Force Microscopy measurements. According to our model, for example, a small conductivity can induce an error of more than two orders of magnitude in the determination of the dielectric constant of a material. Finally, we suggest a method to discriminate between permittivity and conductivity effects by analyzing the dependence of the signal with the tip-sample distance.
\end{abstract}

\section{Introduction}

In recent years, Electrostatic Force Microscopy (EFM) has become a useful tool in quantitative characterization of different properties at the nanoscale [1-6]. These technical improvements have been especially relevant in the characterization of thin films [7-10]. Most of the previous work has been focused on perfectly insulating dielectrics [11], but the possible effect of a small conductivity on the EFM signal has not been addressed before (i.e., previously, every layer included in the sample was only characterized by the thickness and dielectric constant). The conductivity, even with small values, is very important in the characterization of thin films in air ambient conditions where water and molecular adsorption and water condensation [12-14] can be detected as a finite surface conductivity due to the ions added on the otherwise insulating sample. Significant finite conductivity effects are also expected for thin films of conducting materials with a finite length since they cannot accumulate enough free charge in the required regions to compensate external electric fields [15]. The presence of defects on the surfaces is also a factor that implies the presence free charges in certain regions that could change the conductivity of the sample $[16,17]$.

For the discussion of the effects of the thin film conductivity, we are going to introduce the concept of thin film screening length $\lambda$, which is a magnitude directly related to the free charge in the film and can be defined as the maximum length that the electric field is able to penetrate in the sample. This concept is useful in this context because it is a length and it can be directly compared to the length of the thin film itself. For example, conducting thin films, with a screening length much smaller than the film thickness, are relatively easy to characterize since there is a perfect screening of the whole sample composed by the thin film and substrate. This system has been widely studied before $[18,19]$. For this reason, in this paper we will focus on the effects of having thin films with small values of conductivity. In the present paper, we will include a theoretical model that includes the possibility of thin films with certain conductivity, which is a physical effect that was not taken into account in previous related articles [11]. This limit is much more difficult 
to analyse since, due to the relatively large values of the screening length, the electric field is able to penetrate the thin film and the substrate and relevant effects of the electric field distribution must be expected. With the electric field being the most important magnitude in Electrostatic Force Microscopy (EFM), we will analyse the effects of the thin film conductivity on the microscope signal. We will demonstrate that the presence of a small amount of free charge in thin films can be seen as a thin film with an extremely high dielectric constant. This is a very important result since we demonstrated previously that EFM has a very good resolution in those circumstances.

\section{Theoretical Model}

In a typical EFM setup, we have a metallic tip connected to a battery that applies a constant electric potential $V_{0}$. The tip is placed over a sample at a tip-sample distance $D$. The tip usually takes the shape of a cone that is attached to the cantilever in its wider size. In that way, the sharp apex of the tip is placed in the closest size to the sample. It is well known that the macroscopic shape of the tip plays a relevant role in the EFM signal $[20,21]$, especially when the tipsample distance is comparable to other magnitudes such as the tip radius. However, the tip cone shape does not affect the field distribution near the tip apex in the very proximity of the sample [22]. In this paper we will focus on tip-sample distances that are between $0.1 R$ and $0.5 R$. Within this limit and in order to understand the main physics associated with sample's finite conductivity, a simpler tip characterization is adequate. The tip in our simulations is going to be defined by the tip apex radius $R$. The sample conductivity is included through a simple linearized Thomas-Fermi (Debby-Hückel) approximation. In this model, taking into account that $\mathbf{E}(\mathbf{r})=$ $-\nabla V(\mathbf{r})$, the electrostatic potential can be obtained by solving the following expression:

$$
\nabla^{2} V_{i}(r)-\frac{1}{\lambda_{i}^{2}} V_{i}(r)=0,
$$

where we are assuming that $\varepsilon$ is uniform inside each $i$ region and $\lambda$ is the screening length, which is related to the conductivity $\sigma$ by $\lambda^{2}=\left(\varepsilon \varepsilon_{0} D_{\text {diff }}\right) / \sigma$, where $D_{\text {diff }}$ is the diffusion constant of the material and $\varepsilon$ is the thin film dielectric constant.

In the case of a thin film with finite nonzero conductivity, the sample is going to be composed of two layers, as shown in Figure 1: (1) a thin film with dielectric constant $\varepsilon$, thickness $h$, and screening length $\lambda$ and (2) a dielectric substrate with dielectric constant $\varepsilon_{2}$. When the sample does not have any conductivity (i.e., $\lambda=\infty$ ) the electrostatic potential of a punctual charge is obtained by the image charge theory, where the image charges are usually obtained by solving the Laplace/Poisson equation in cylindrical coordinates $(z, \rho, \phi)$.

From the geometry described in the previous paragraph, we only have $\lambda \neq 0$ inside the thin film. For any other region, $\lambda_{i}=\infty$ and (1) becomes again the Poisson/Laplace equation. Following the scheme shown in Figure 1(d), for every $i$ region, the electrostatic potential $V_{i}(z, \rho)$, for a single point charge $q$ located in region $i=0$ at $\left(z_{q}=0, \rho=0\right)$, can be written as

$$
\begin{aligned}
& V_{i} \\
& \quad=\frac{q}{4 \pi \varepsilon_{0}}\left[\int_{0}^{\infty} J_{0}(k \rho)\left(\psi_{i}(k) e^{-K_{z} z}+\theta_{i}(k) e^{K_{z} z}\right) d k\right],
\end{aligned}
$$

where $K_{z}^{2}=k^{2}-1 / \lambda_{i}^{2}, J_{0}$ are the first-order Bessel functions, and $\psi_{i}$ and $\theta_{i}$ are coefficients obtained by applying the electrostatic boundary conditions $\left(V_{i}=V_{i+1}\right.$ and $\varepsilon_{i} V_{i}^{\prime}=$ $\varepsilon_{i+1} V_{i+1}^{\prime}$ at $\left.z=z_{i, i+1}\right)$ to the sample interfaces. In the region where the punctual charge is placed $(i=0)$, we have $\psi_{0}=1$. The other coefficients take the following form:

$$
\begin{aligned}
& \widetilde{H} \theta_{0}(K)=\left(C_{12} e^{-2\left(k-K_{z}\right) a}\right) e^{-2 K_{z} b}-C_{1} e^{-2 k a}, \\
& \widetilde{H} \theta_{1}(K)=\frac{2 C_{12}}{\varepsilon_{1}+k / K_{z}} e^{-\left(k-K_{z}\right) a} e^{-2 K_{z} b}, \\
& \widetilde{H} \psi_{1}(K)=\frac{2}{\varepsilon_{1}+k / K_{z}} e^{-\left(k-K_{z}\right) a}, \\
& \widetilde{H} \psi_{2}(K)=\frac{4 \varepsilon_{1} k / K_{z}}{\left(\varepsilon_{1}+k / K_{z}\right)\left(\varepsilon_{1}+\varepsilon_{2} k / K_{z}\right)} e^{\left(k-K_{z}\right)(b-a)},
\end{aligned}
$$

where $a$ and $b$ are described in Figure $1(\mathrm{~d})$ and $\widetilde{H}$ can be defined as

$$
\widetilde{H}=1-C_{12} C_{1} e^{-2 K_{z} h},
$$

where $C_{1}=\left(\varepsilon_{1}-k / K_{z}\right) /\left(\varepsilon_{1}+k / K_{z}\right)$ and $C_{12}=\left(\varepsilon_{1}-\right.$ $\left.\varepsilon_{2} k / K_{z}\right) /\left(\varepsilon_{1}+\varepsilon_{2} k / K_{z}\right)$. Equation (2) is the general form of the electrostatic potential obtained in cylindrical coordinates when the Laplace/Poisson equation is being solved. This solution is the most adequate for geometries like the one in this paper (see Figure 1) since the cylindrical symmetry around the $z$-axis allows us to easily obtained the specific solution of the electrostatic potential at any region by applying the electrostatic boundary conditions. Equations (3) and (4) give the exact values of the coefficients from regions 0 to 2 , which are the only parameters that are unknown in (2). The same procedure can be done for any other geometry with the same axial symmetry. However, for geometries with more than 3 regions, the exact analytical values for the coefficients $\theta_{i}$ and $\psi_{i}$ become hard to obtain and manage. In these cases, numerical methods such as the transfer or scattering matrix methods, which define the relation between the coefficients of different interfaces by a numerical matrix, have been used successfully in the past [23]. It is worth noting that the forms of (3) and (4) for the particular case of $\lambda_{i}=\infty$ have been previously described in [11]. The generalization of this procedure to the particular cases when there is certain conductivity in the thin film $\left(\lambda_{i} \neq \infty\right)$ is one of the main contributions of this paper. These results can be also useful to improve the quantitative description of the conductivity of samples such as graphene layers, where it has been demonstrated that their conductivity can be measured (or at least estimated) by EFM [15]. 


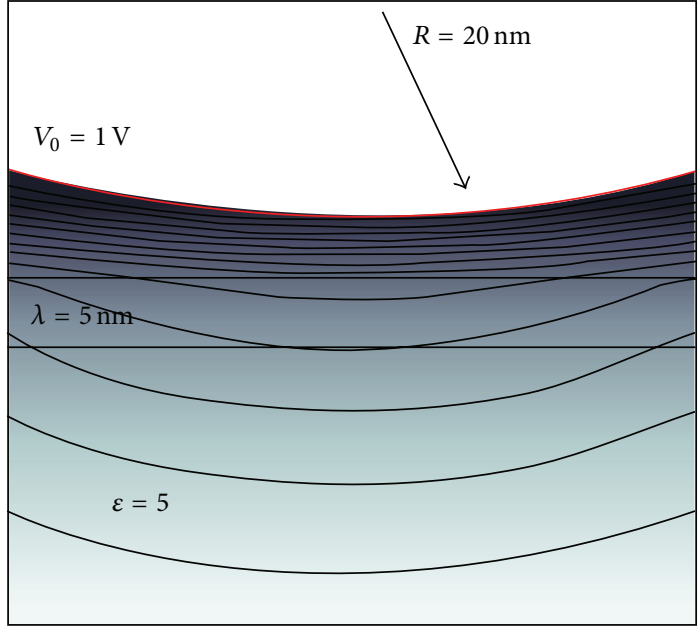

(a)

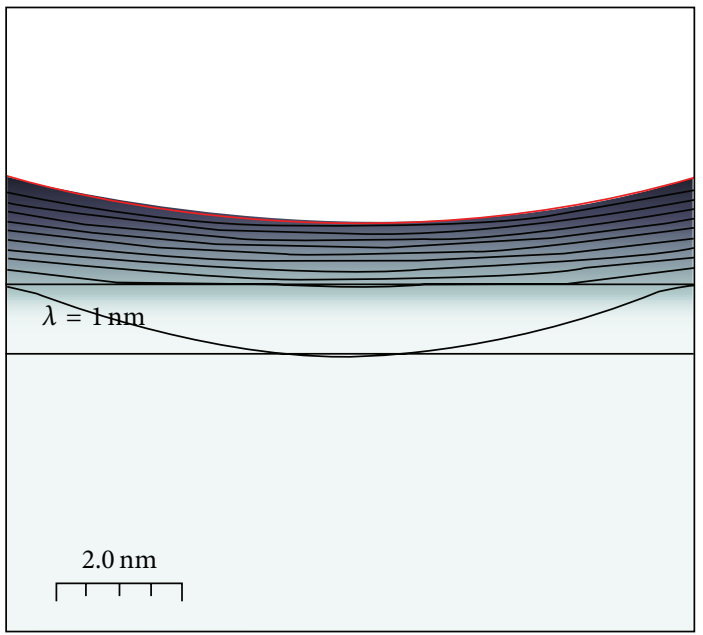

(c)

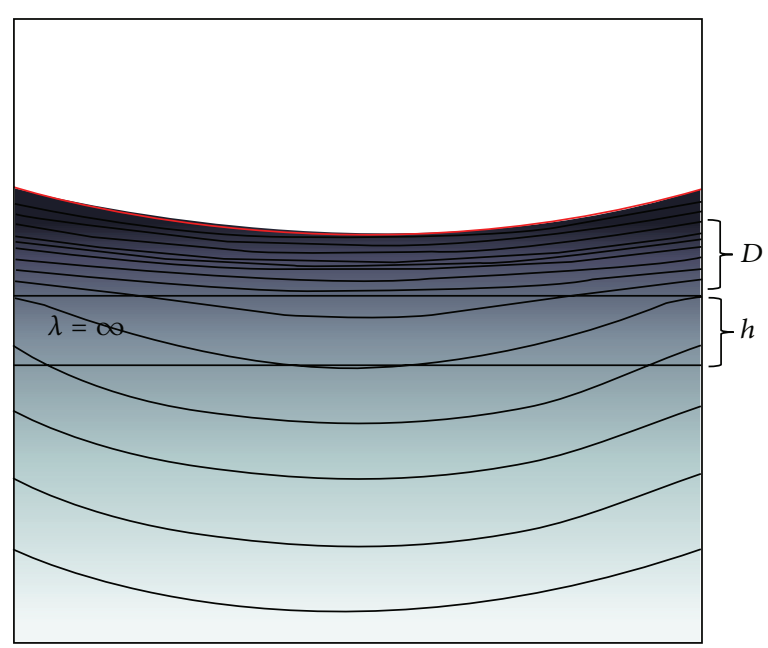

(b)

\begin{tabular}{ccc}
$i=0$ & Air & $z=a$ \\
\hline$i=1$ & Film & $z=b$ \\
\hline$i=2$ & Substrate &
\end{tabular}

(d)

FIGURE 1: Equipotential distribution of the tip-sample system in Electrostatic Force Microscopy for 3 different thin film screening lengths. The arrow shown in the potential distribution shows the region that has been simulated.

To solve electrostatic problems with this geometry, an algorithm called the Generalized Image Charge Method (GICM) has been developed [20]. The GICM replaces the surface charge density by a set of charges inside the metallic tip and the sample by a set of image charges. The $q_{i}$ charge values are obtained by a standard least-squares minimization (LSM). Once the charge distribution is obtained by the tip, the electrostatic force $F(\varepsilon, \lambda)$ can be directly obtained by the interaction between the tip charges and their images.

\section{Effects of Small Thin Film Conductivities}

In Figure 1 we show three different equipotential distributions that only differ in the $\lambda$ value of the thin film. The EFM setup has been simulated by $R=20 \mathrm{~nm}, D=1 \mathrm{~nm}, h=1 \mathrm{~nm}$, and $V_{0}=1 \mathrm{~V}$. For simplicity, we have fixed the dielectric constant of both film and substrate to $\varepsilon=\varepsilon_{2}=5$ (i.e., the only difference between film and substrate in Figure 1 is the screening length $\lambda$ ). When $\lambda=\infty$, the thin film-substrate system becomes a semi-infinite dielectric sample with $\varepsilon=5$, which corresponds to the equipotential distribution shown in Figure $1(\mathrm{~b})$. As it can be seen, smaller $\lambda$ values imply a smaller electric field inside the sample, as it should be. This effect is also found when the thin film dielectric constant increases, as shown in Figure 2(a) where we plot the electrostatic potential drop along the $z$-axis (from the tip apex to the substrate below the film) for the equipotential distributions shown in Figure 1. To compare the effect of having a nonzero conductivity (i.e., finite $\lambda$ ) and having a higher $\varepsilon$ value in the thin film, we also show the electrostatic potential drop for $(\lambda=\infty, \varepsilon=110)$ and $(\lambda=\infty, \varepsilon=500)$. These results are particularly relevant since they show that different combinations of dielectric constant and screening length can give rise to the same electrostatic force; for example, $F(\varepsilon=5, \lambda=5 \mathrm{~nm})=$ $F(\varepsilon=110, \lambda=\infty)$ at $D=1 \mathrm{~nm}$ and $F(\varepsilon=5, \lambda=5 \mathrm{~nm})=$ $F(\varepsilon=500, \lambda=\infty)$ at $D=10 \mathrm{~nm}$, as we show in Figure 2(b). Comparing the potential drop of these three samples, we see 


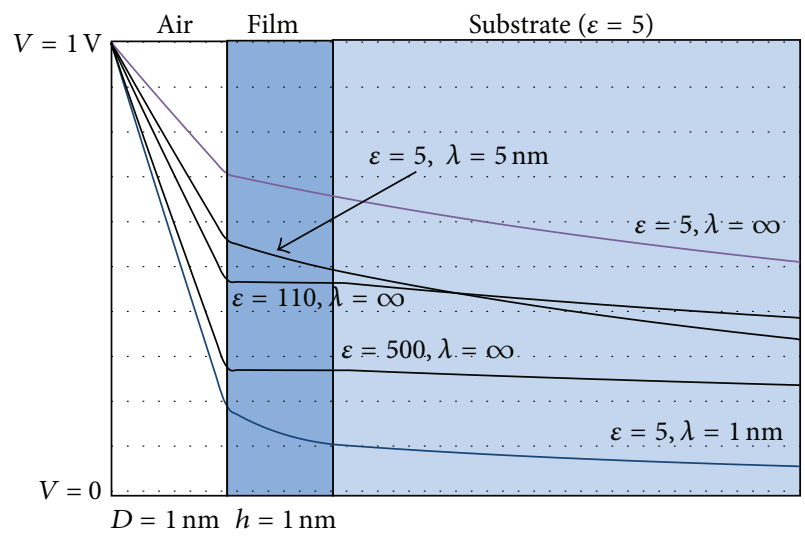

(a)

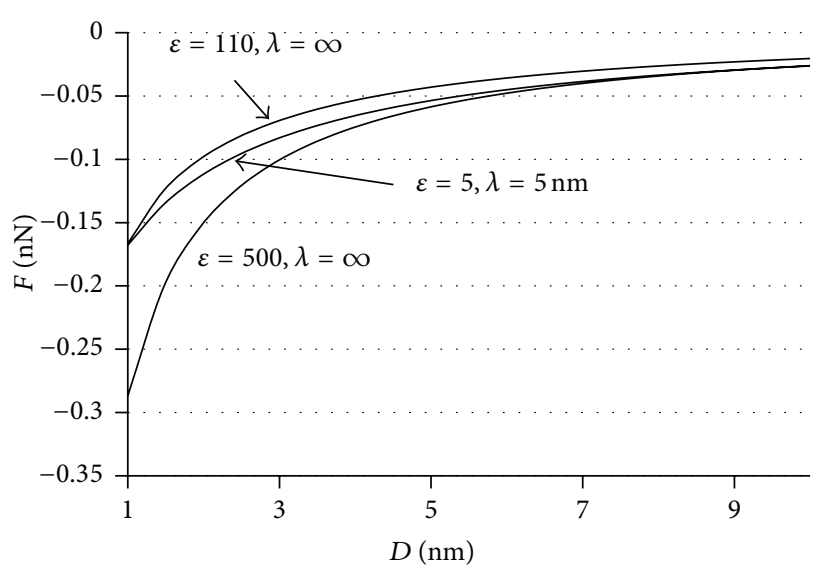

(b)

FIGURE 2: (a) Electrostatic potential drop between the tip apex and the sample for 5 thin film configurations, described in the figure by $\varepsilon$ and $\lambda$. (b) Electrostatic vertical force versus tip-sample distance for three related thin film configurations, where the dielectric constants of the thin films that do not have any conductivity have been adjusted to fit the upper and lower limit of the thin film with $\lambda=5 \mathrm{~nm}$. In both figures, $R=20 \mathrm{~nm}, \varepsilon_{2}=5, V_{0}=1 \mathrm{~V}$, and $h=1 \mathrm{~nm}$.

that finite $\lambda$ and high $\varepsilon$ values reduce the electric field inside the sample. However, they have very different effects on the electrostatic potential and on the electric field distribution. Focusing on these three curves, we see that when $\lambda=5 \mathrm{~nm}$, the electric field (potential gradient) is lower in the region near to the tip (air or vacuum) and higher inside the sample, compared to the cases with high $\varepsilon$. It is worth noting that as we can see in Figure 2(b), the effects of conductivity can be also distinguishable for $D$ values higher than $1 \mathrm{~nm}$. This fact makes the method useful even in air condition, where the minimum working tip-sample distance is limited by the jump into contact distance. In this paper we have assumed $D=1 \mathrm{~nm}$ in most of the cases for clarity in the figures, but higher $D$ values could be also used.

In Figure 3 we show $F$ as a function of $\lambda$ (Figure 3(a)) and $\varepsilon$ (Figure 3(b)) for two different tip-sample distances $(D=$ $1 \mathrm{~nm}$ and $D=10 \mathrm{~nm}$ ). Focusing on $D=1 \mathrm{~nm}$, we see that $F$ does not converge until $\lambda>20$ or $\varepsilon>40000$. This implies that when working at very small tip-sample distances and small film thicknesses, the EFM is able to distinguish huge $\varepsilon$ values, as it has been reported before [24]. Due to the small thin film thickness, the electric field is able to go through the film and penetrate in the substrate. For higher $h$ or $D$ values, the electric field vanishes inside the film and no difference can be effectively found between a thin film and a semi-infinite metallic sample. The values of the screening length that can be distinguished also imply that small amounts of conductivity are able to change the electrostatic signal significantly. This fact makes extremely important having under control any possible presence of conductivity in the sample, especially when quantitative magnitudes are being measured. Another parameter that must be taken into account is the tip-sample distance. As we can see in Figure 3, $F(D=10 \mathrm{~nm})$ converges much faster than $F(D=1 \mathrm{~nm})$. This fact makes the problem even harder since the effect of having a finite screening length is different for different tip-sample distances. Moreover, as we can see in Figure 2(b), $F(\lambda=5 \mathrm{~nm})=F(\varepsilon=110$, $\lambda=\infty$ ) when $D=1 \mathrm{~nm}$. However, both curves diverge when $D$ becomes higher. On the upper limit under study $(D=10 \mathrm{~nm})$, the $\varepsilon$ value that corresponds to $F(\lambda=5 \mathrm{~nm})$ is $\varepsilon=500$. In other words, the presence of conductivity can be confused with insulating thin films characterized by dielectric constants that differ around an order of magnitude, depending on the working tip-sample distance.

In Figure 4 we show the combinations of $\varepsilon$ and $\lambda$ that gives the same EFM signals for $D=1$ and $10 \mathrm{~nm}$. As we can see, there is a big window of $\varepsilon$ values that, depending on the working tip-sample distance, are equivalent to a certain $\lambda$ value. The window of possible $\varepsilon$ values becomes wider when $D$ becomes smaller. For example, the $\varepsilon$ values go from $\varepsilon=110$ to $\varepsilon=500$ when $\lambda=5 \mathrm{~nm}$ and from $\varepsilon=2500$ to $\varepsilon=20000$ when $\lambda=1 \mathrm{~nm}$. Although this fact can be assumed as inconvenient at the first sight, it can be also used to distinguish the physical phenomena involved in the EFM signal by measuring the electrostatic force at two different tipsample distances. If the apparent dielectric constant of the material differs significantly, we may think that the sample is having a conductive behavior.

It is worth noting that when working at very small tipsample distances, van der Waals and water meniscus forces may have an influence in the final results and must be taken into account or avoided. In the case of van der Waals force, increasing the tip-sample distance is a good method to avoid its effect since it has a stronger dependence with the distance than the electrostatic forces. In the case of water meniscus, it is better to reduce the tip voltage since their formation is directly related to the electrostatic energy (proportional to the tip voltage) [25].

\section{Conclusions}

In conclusion, we have developed a method to simulate and study the effects of the conductivity in thin film samples. We 


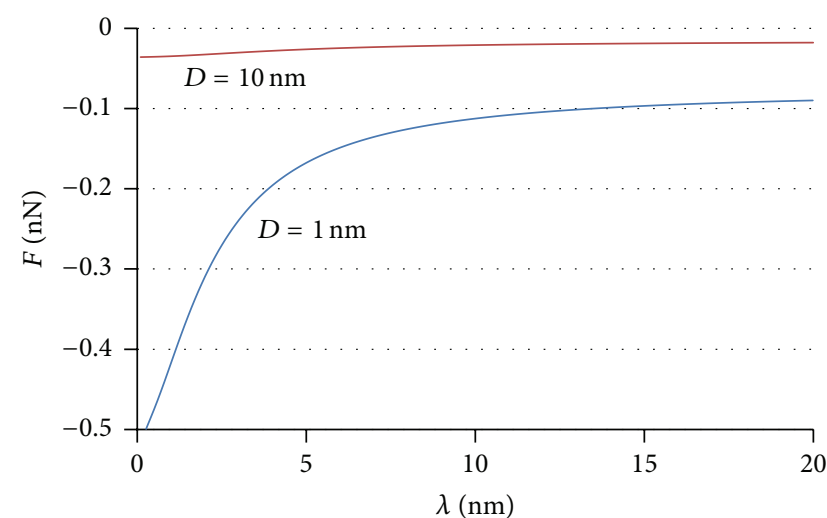

(a)

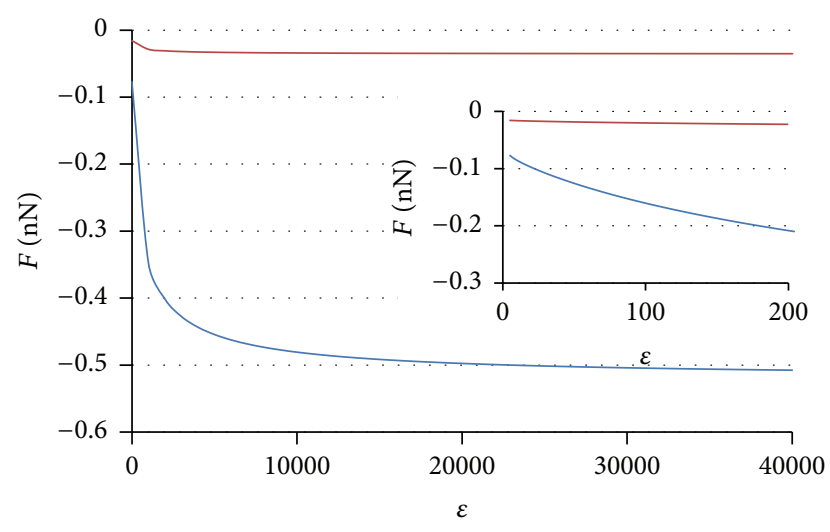

(b)

Figure 3: (a) Vertical electrostatic force as a function of the screening length. (b) Vertical electrostatic force as a function of the thin film dielectric constant. Inset shows the values of the force for smaller dielectric constants. In both figures, the force has been calculated for $D=1 \mathrm{~nm}$ and $D=10 \mathrm{~nm}$, and $R=20 \mathrm{~nm}, h=1 \mathrm{~nm}, \varepsilon_{2}=5$, and $V_{0}=1 \mathrm{~V}$.

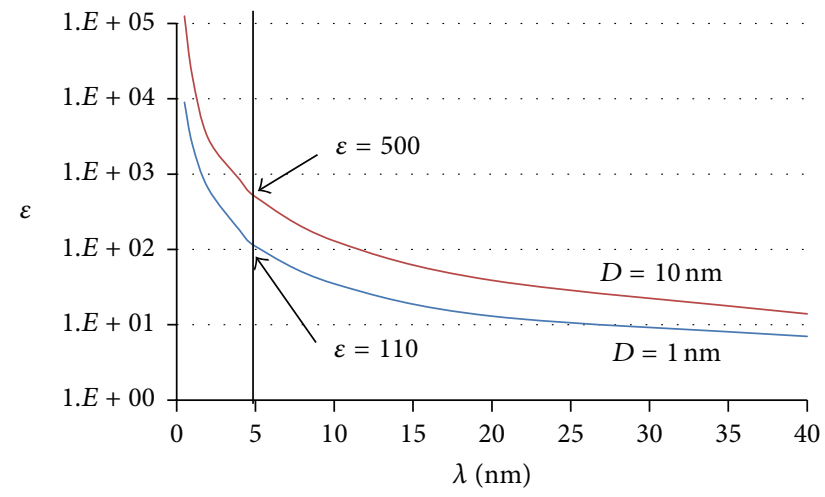

FIGURE 4: Relation between the dielectric constant of nonconductive thin films and the screening length of thin films with $\varepsilon=5$, and $R=20 \mathrm{~nm}, h=1 \mathrm{~nm}, \varepsilon_{2}=5$, and $V_{0}=1 \mathrm{~V}$.

have demonstrated that very small values of conductivity can change the electrostatic force in EFM to values similar to those obtained from thin films with extremely high dielectric constants. For example, a thin film with $\lambda=5 \mathrm{~nm}$ and $\varepsilon=$ 5 can be compared to thin films without any conductivity and $\varepsilon$ between 110 and 500 . We have also demonstrated that the equivalent $\varepsilon$ value can change more than one order of magnitude depending on the working tip-sample distance. The strong dependence with the tip-sample distance can be used to distinguish the origin of the electrostatic signal since, only in the case of having conductivity, different $\varepsilon$ values would be measured at different distances.

\section{Conflict of Interests}

The authors declare that there is no conflict of interests regarding the publication of this paper.

\section{Acknowledgments}

The authors acknowledge J. J. Sáenz, C. Gómez-Navarro, and J. Gómez-Herrero for insightful discussions. This work has been partially funded by the Banco Santander-UAM Research Program. G. M. Sacha acknowledges support from the Spanish Ramón y Cajal Program.

\section{References}

[1] R. I. Revilla, X.-J. Li, Y.-L. Yang, and C. Wang, "Comparative method to quantify dielectric constant at nanoscale using atomic force microscopy," The Journal of Physical Chemistry C, vol. 118, no. 10, pp. 5556-5562, 2014.

[2] G. M. Sacha and P. Varona, "Artificial intelligence in nanotechnology," Nanotechnology, vol. 24, no. 45, Article ID 452002, 2013.

[3] S. V. Kalinin, S. Jesse, B. J. Rodriguez, E. A. Eliseev, V. Gopalan, and A. N. Morozovska, "Quantitative determination of tip parameters in piezoresponse force microscopy," Applied Physics Letters, vol. 90, no. 21, Article ID 212905, 2007.

[4] S. Guriyanova, D. S. Golovko, and E. Bonaccurso, "Cantilever contribution to the total electrostatic force measured with the atomic force microscope," Measurement Science and Technology, vol. 21, no. 2, Article ID 025502, 2010.

[5] E. Palacios-Lidón, J. Abellán, J. Colchero, C. Munuera, and C. Ocal, "Quantitative electrostatic force microscopy on heterogeneous nanoscale samples," Applied Physics Letters, vol. 87, no. 15, Article ID 154106, 2005.

[6] S. F. Lyuksyutov, R. A. Vaia, P. B. Paramonov et al., "Electrostatic nanolithography in polymers using atomic force microscopy," Nature Materials, vol. 2, no. 7, pp. 468-472, 2003.

[7] Y. Fan, G. Hao, S. Luo et al., "Synthesis, characterization and electrostatic properties of $\mathrm{WS}_{2}$ nanostructures," AIP Advances, vol. 4, Article ID 057105, 2014.

[8] Y. Shen, Y. Wang, J. Zhang et al., "Sample-charged mode scanning polarization force microscopy for characterizing reduced graphene oxide sheets," Journal of Applied Physics, vol. 115, no. 24, Article ID 244302, 2014. 
[9] E. M. Vogel, "Technology and metrology of new electronic materials and devices," Nature Nanotechnology, vol. 2, no. 1, pp. 25-32, 2007.

[10] E. Escasaín, E. López-Elvira, A. M. Baró, J. Colchero, and E. Palacios-Lidon, "Nanoscale electro-optical properties of organic semiconducting thin films: from individual materials to the blend," The Journal of Physical Chemistry C, vol. 116, no. 33, pp. 17919-17927, 2012.

[11] E. Castellano-Hernández, J. Moreno-Llorena, J. J. Sáenz, and G. M. Sacha, "Enhanced dielectric constant resolution of thin insulating films by electrostatic force microscopy," Journal of Physics: Condensed Matter, vol. 24, no. 15, Article ID 155303, 2012.

[12] L. Xu and M. Salmeron, "Scanning polarization force microscopy study of the condensation and wetting properties of glycerol on mica," The Journal of Physical Chemistry B, vol. 102, no. 37, pp. 7210-7215, 1998.

[13] L. Xu, A. Lio, J. Hu, D. F. Ogletree, and M. Salmeron, "Wetting and capillary phenomena of water on mica," The Journal of Physical Chemistry B, vol. 102, no. 3, pp. 540-548, 1998.

[14] F. Rieutord and M. Salmeron, "Wetting properties at the submicrometer scale: a scanning polarization force microscopy study," The Journal of Physical Chemistry B, vol. 102, no. 20, pp. 3941-3944, 1998.

[15] C. Gómez-Navarro, F. J. Guzmán-Vázquez, J. Gómez-Herrero, J. J. Saenz, and G. M. Sacha, "Fast and non-invasive conductivity determination by the dielectric response of reduced graphene oxide: an electrostatic force microscopy study," Nanoscale, vol. 4, no. 22, pp. 7231-7236, 2012.

[16] S. R. Bishop, H. L. Tuller, G. Ciampi et al., "The defect and transport properties of acceptor doped TlBr: role of dopant exsolution and association," Physical Chemistry Chemical Physics, vol. 14, no. 29, pp. 10160-10167, 2012.

[17] J. Engel and H. L. Tuller, "The electrical conductivity of thin film donor doped hematite: from insulator to semiconductor by defect modulation," Physical Chemistry Chemical Physics, vol. 16, no. 23, pp. 11374-11380, 2014.

[18] S. Belaidi, P. Girard, and G. Leveque, "Electrostatic forces acting on the tip in atomic force microscopy: modelization and comparison with analytic expressions," Journal of Applied Physics, vol. 81, no. 3, pp. 1023-1030, 1997.

[19] S. Gomez-Monivas, L. S. Froufe-Perez, A. J. Caaman, and J. J. Saenz, "Electrostatic forces between sharp tips and metallic and dielectric samples," Applied Physics Letters, vol. 79, no. 24, article 4048, 2001.

[20] G. M. Sacha, E. Sahagún, and J. J. Sáenz, "A method for calculating capacitances and electrostatic forces in atomic force microscopy," Journal of Applied Physics, vol. 101, no. 2, Article ID 024310, 2007.

[21] G. M. Sacha, M. Cardellach, J. J. Segura et al., "Influence of the macroscopic shape of the tip on the contrast in scanning polarization force microscopy images," Nanotechnology, vol. 20, no. 28, Article ID 285704, 2009.

[22] J. Colchero, A. Gil, and A. M. Baró, "Resolution enhancement and improved data interpretation in electrostatic force microscopy," Physical Review B, vol. 64, no. 24, Article ID 245403, 2001.

[23] G. M. Sacha and J. J. Sáenz, "Generalized scattering matrix method for electrostatic calculations of nanoscale systems," Physical Review B, vol. 77, no. 24, Article ID 245423, 2008.

[24] E. Castellano-Hernández and G. M. Sacha, "Ultrahigh dielectric constant of thin films obtained by electrostatic force microscopy and artificial neural networks," Applied Physics Letters, vol. 100, no. 2, Article ID 023101, 2012.

[25] S. Gómez-Moñivas, J. J. Sáenz, M. Calleja, and R. García, "Fieldinduced formation of nanometer-sized water bridges," Physical Review Letters, vol. 91, no. 5, Article ID 056101, 2003. 

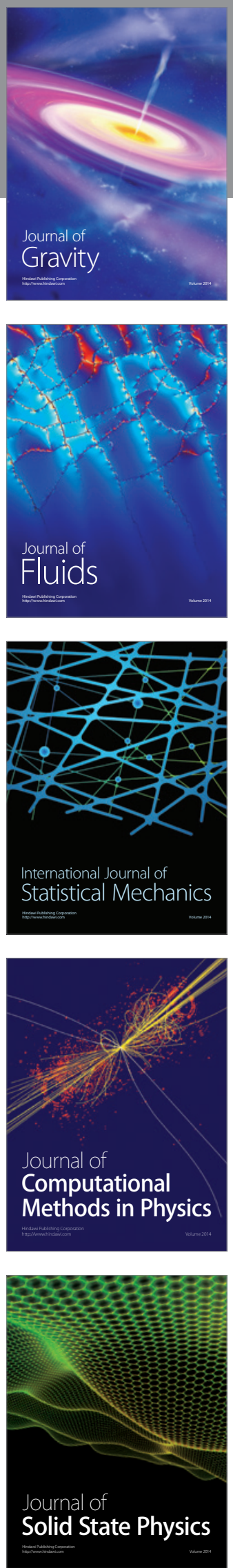

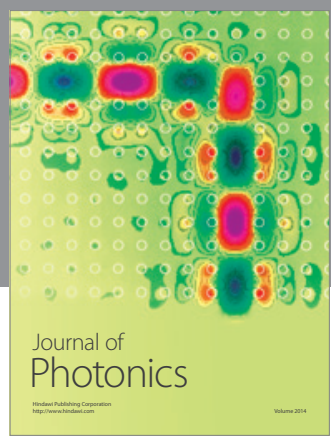

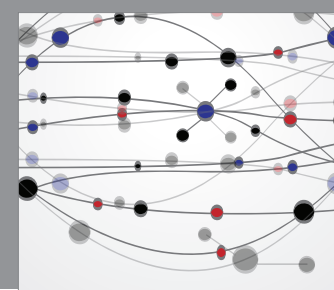

The Scientific World Journal

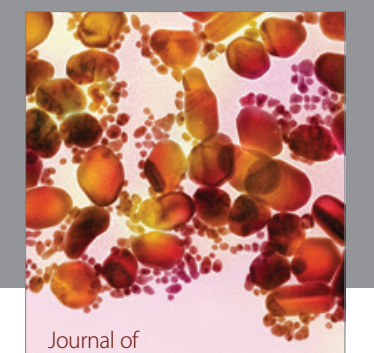

Soft Matter
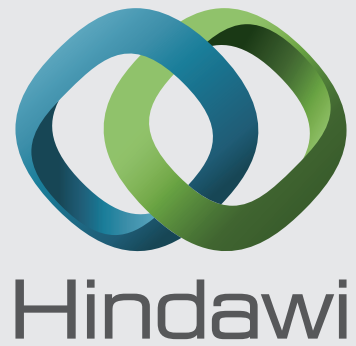

Submit your manuscripts at

http://www.hindawi.com
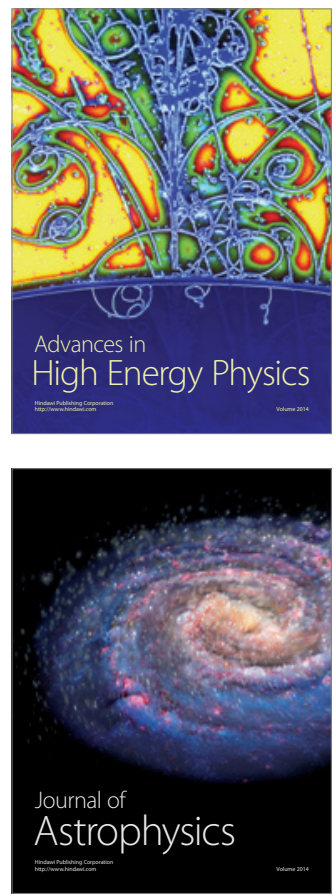
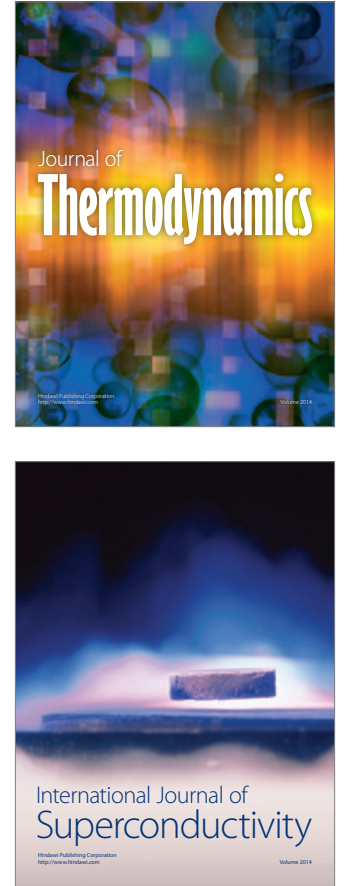
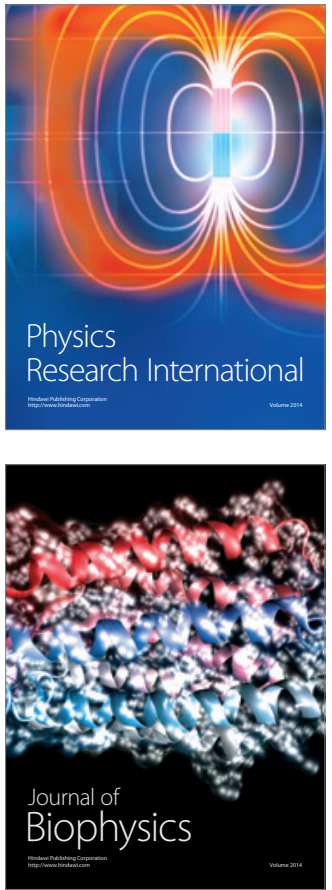
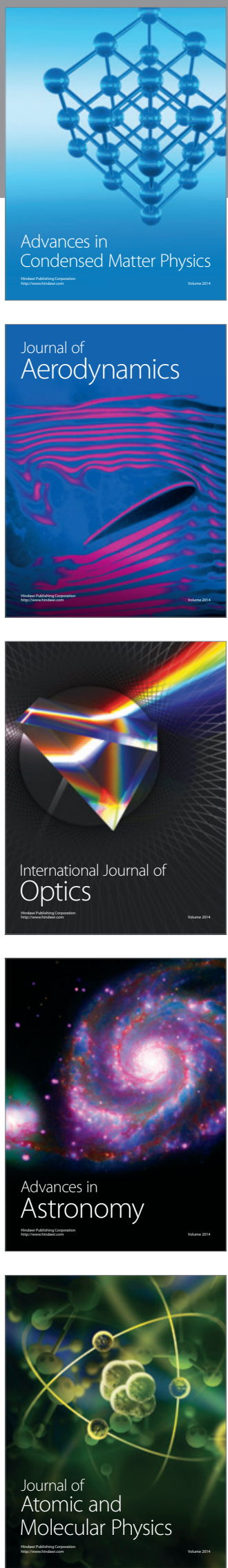